\title{
Research Progress of Fire Detection Based on Remote Sensing
}

\author{
Wang Shidong \\ 1. College of Resources Science \& Technology, Beijing \\ Normal University, Beijing, China, 100875 \\ 2. School of Surveying and Land Information \\ Engineering, Henan Polytechnic University, Jiaozuo, \\ China, 454000
}

\author{
Zhang Hebing \\ School of Surveying and Land Information Engineering \\ Henan Polytechnic University \\ Jiaozuo, China, 454000
}

\begin{abstract}
In this paper, the principle of forest fire detection was discussed firstly, and on the basis, the research progress of forest fire detection based on AVHRR AND MODIS was discussed at home and abroad. In general, the AVHRR and MODIS data are used as the main tools for forest fire detection. In recent years, many scholars developed a variety of forest fire detection algorithms based on AVHRR AND MODIS data that were tested and applied in practice and achieved good results. According to the characteristics of environment and disaster monitoring small satellite, the existing contextual algorithm based on MODIS is improved to adapt to HJ-IRS. The process of improved forest fire detection algorithm includes potential fire spots judgment, absolute fire spots judgment, contextual analysis, relative fire spots judgment and confidence degree of fire spots.
\end{abstract}

Keywords- AVHRR, MODIS, Fire detection, HJ, Remote sensing

\section{INTRODUCTION}

Forest fires have drawn increasing attention in recent years due to their tremendous effects on environment, humans and wildlife, ecosystem function, weather, and climate. Accurate monitoring and mapping of the spatial and temporal distribution of forest fires is important since it contributes in fire effects assessing and controlling, and a number of ongoing studies, like land use, land-cover change, and climate change.

In most cases, a small fire cannot be detected and stopped timely and lead to a big fire finally. Therefore, it is very important to protect ecological environment and forest resources to monitor forest fires accurately and timely. In the past, it is costly and has low accuracy to monitor forest fires relying on manpower and aircraft. In recent years, the technology of satellite remote sensing becomes a powerful tool for monitoring forest fires accurately and timely.

Since the 1980's, beginning to study fire detection using satellite data, many scholars at home and abroad carried out research on the methods of forest fires monitoring for different sensors. In general, the NOAA/AVHRR and MODIS data are used as the main tools for forest fire detection. In recent years, both domestic and foreign scholars developed a variety of forest fire detection algorithms based on remote sensing data that were tested and applied in practice and achieved good results.

\section{PRINCIPLE Of Forest Fire DeteCtion}

The principle of forest fire detection is to identify the fire pixels according to the temperature of ignition spot is higher than ambient temperature. The basis of judgment is the relationship of heat radiation degree between temperature and wavelength [1]. The radiation energy of any object is a function of surface temperature. The Stefan-Boltzmann law shows the property, as follows:

$\mathrm{M}(\mathrm{T})=\sigma \mathrm{T} 4$

Where, $\mathrm{M}(\mathrm{T})$ represents the surface emission energy of blackbody, namely total radiant exitance (W.m-2); $\sigma$ is the Stefan-Boltzmann constant which is $5.6697 \times 10-8 \mathrm{~W} \cdot \mathrm{m}-2 \cdot \mathrm{K}-$ 4 ; $\mathrm{T}$ is the thermodynamic temperature of emitters, namely blackbody temperature $(\mathrm{K})$.

The Stefan-Boltzmann law states that the total power (energy per unit time) emitted by a blackbody, per unit surface area of the blackbody, varies as the fourth power of the temperature. That is to say, a subtle change of temperature will cause a great change in radiation. The temperature of forest fire and other heat source will cause more dramatic changes in radiation, which is very beneficial to judge the high-temperature heat source. The emission energy of objects changes with temperature and wavelength. The Wien's displacement law states that the wavelength at which the blackbody emission spectrum is most intense varies inversely with the blackbody's temperature, as follows:

\section{$\lambda \max =\mathrm{A} / \mathrm{T}$}

Where, $\lambda \max$ is the maximum wavelength of radiation intensity which its unit is $\mu \mathrm{m}$; $\mathrm{A}$ is the constant which is $2898 \mu \mathrm{m} \cdot \mathrm{K}$; $\mathrm{T}$ is the thermodynamic temperature $\mathrm{K}$.

The Wien's displacement law shows that the distribution of radiation energy peak will move to the shorter wavelength as temperature becomes higher, that is to say, if the temperature becomes higher, the wavelength of radiation peak will become shorter. The wavelength of ground surface radiation peak in normal temperature (about $300 \mathrm{~K}$ ) is about $10 \mu \mathrm{m}$. While the flame temperature is generally above 500 $700 \mathrm{~K}$ and its wavelength of thermal radiation peak is about $3-5 \mu \mathrm{m}$. The flame temperature of forest fires can reach about $1000 \mathrm{~K}$, and its wavelength of thermal radiation peak will become less than $3 \mu \mathrm{m}$. For blackbody radiation source, the Planck's formula shows the relationship of radiation intensity with temperature and wavelength [2]. According to the Planck's formula, the radiation energy of high temperature spots is larger in middle-infrared band than thermal infrared 
band. Therefore, the middle-infrared band is more sensitive to high temperature spots than thermal infrared band. The basic principle of fire detection is that gets the difference of brightness temperature $\triangle \mathrm{T}$ between $4 \mu \mathrm{m}$ and $11 \mu \mathrm{m}$ bands.

\section{Forest FIRE DETECTION BASE ON AVHRR}

The advanced very high resolution radiometer (AVHRR) is a space-borne sensor embarked on the National Oceanic and Atmospheric Administration (NOAA) family of polar orbiting platforms. AVHRR instruments measure the reflectance of the Earth in 5 relatively wide spectral bands. NOAA has at least two polar-orbiting meteorological satellites in orbit at all times. The primary sensor on board both satellites is the AVHRR instrument. Morning-satellite data are most commonly used for land studies, while data from both satellites are used for atmosphere and ocean studies. Together they provide twice-daily global coverage, and ensure that data for any region of the earth are no more than six hours old. The swath width, the width of the area on the Earth's surface that the satellite can "see", is approximately 2,500 kilometers. The highest ground resolution that can be obtained from the current AVHRR instruments is 1.1 kilometer. Remote sensing applications of the AVHRR sensor are based on validation techniques of colocated ground observations and satellite observations. Alternatively, radiative transfer calculations are performed. There are specialized codes which allow simulation of the AVHRR observable brightness temperatures and radiances in near infrared and infrared channels.

In recent years, AVHRR data have been widely used in monitoring forest fires, and a number of methods of fire detection based on AVHRR data were developed, as follows: Kaufman detected fire spots using a fixed threshold method [3]; Flasse detected fire spots using an adjacent pixels analytical method [4]; Chuvieco remove the area that is not vegetation by limiting the threshold of normalized difference vegetation index (NDVI) in order to reduce misjudgments [5]; Boles compared three methods of monitoring forest fires based on AVHRR data taking Alaska as an example [6]; Dozier developed a method of detection high-temperature spots based on NOAA/AVHRR sub-pixels [7]. In a word, monitoring fire based on AVHRR data has made some achievements. But because of its lower radiation resolution, the thermal infrared band at about $325 \mathrm{~K}$ will reach saturation and is unsuitable for detection high-temperature spots. Furthermore, the AVHRR only has five bands and cannot verify each other between each band.

\section{FOREST Fire DETECTION BASED ON MODIS}

The moderate-resolution imaging spectroradiometer (MODIS) is an important sensor that is equipped on EOS satellite. The MODIS instrument is operating on both the Terra and Aqua spacecraft. It has a viewing swath width of $2,330 \mathrm{~km}$ and views the entire surface of the Earth every one to two days. MODIS is one of the five sensors on-board TERRA/AQUA satellites with 36 spectral bands and acquires data in three different spatial resolutions $250 \mathrm{~m}$ (bands 1 and 2), 500m (bands 3-7) and 1000m (bands 8-36) covering visible, near infrared, shortwave infrared and thermal-IR regions of the electro magnetic spectrum. TERRA MODIS satellite data for four dates were used in generating daytime fire products using regional thresholds. Therefore, MODIS data have more advantages over AVHRR data for monitoring forest fires. In recent years, the research on forest fire detection based on MODIS has got great attention.

The research team led by Kaufman studied and simulated fire detection algorithms based on MODIS and carried out some experiments in Brazil forests and Africa forests respectively [3]. Some Chinese research institutes and universities also conducted research on application of forest fire detection using MODIS data. For example, Qin Xianlin established the forest fire detection models based on MODIS using brightness temperature vegetation index method [8]; Liang Yun detected many forest fires successfully in Daxinganling forest in 2002 using infrared channel and three-channel synthesis method of MODIS [9]; Liu Liangming studied monitoring effect for a fire in Daxinganling forest using MODIS data and got good results [10]; Zhang Shuyu studied the straw burning status in Guanzhong using MODIS data [11]; Zhou Xiaocheng tested and analyzed fire detection theoretical algorithm based on MODIS for 9 forest fires in China [12]; Huang Jing developed a quick and easy fire detection method through setting the threshold value of $\operatorname{ch} 21$ and $\operatorname{ch} 21-\operatorname{ch} 31$ which was applied in forest fire in Hubei province [13]; Peng Guangxiong developed a method to identify forest fire based on smoke plumes masking by using MODIS data which was applied in Malaysian tropical rainforests and achieved good results[14,15].

The principles of fire detection based on MODIS is that establish the appropriate models to automatically extract fire spots information using the brightness temperature of $4 \mu \mathrm{m}$ and $11 \mu \mathrm{m}$ bands according to difference between background radiation and forest fires radiation [16-20]. At present, the contextual algorithm is a popular approach to detect forest fire by using MODIS data. This method increased the sensitivity of small fire spots detection through setting background threshold of potential fire spots and calculating dynamic fire spots threshold based on contextual spatial analysis, and adding many solar reflective bands in order to remove false fire spots information.

The MODIS contextual algorithm is composed of three basic parts, including preliminary thresholds to identify potential fire pixels, contextual tests to confirm fires among the potential fire pixels [3], and thresholds to reject false alarms. In the first part, the selection of fixed thresholds is subtle as an over-high setting runs a risk of omitting fire pixels. Meanwhile, an over-low setting causes more noise in deriving the parameters of the background pixels [21-25] and generates more false alarms. The MODIS version 4 contextual algorithm employs fixed thresholds globally to identify potential fire pixels. For global applications, the preliminary thresholds cannot be set low enough to detect most small fires that can be physically detected for regional concern. Therefore, it needs improvement for fire monitoring and management at the regional scale. 


\section{FOREST FIRE DETECTION BASED ON ENVIRONMENT} AND DISASTER MONITORING SMALL SATELLITE DATA

At present, in monitoring forest fires by using remote sensing, AVHRR and MODIS data are main tools of forest fire detection, but because the spatial resolution of these sensors is low, lots of small area forest fire spots often cannot be detected, causing not fighting fires timely and becoming campaign fire. The environment and disaster monitoring small satellite has more advantages than AVHRR and MODIS, so can be used to monitor forest fires. However, the existing forest fire detection algorithms based on AVHRR and MODIS can't be directly used in HJ sensor, need be improved according to characteristics of environment and disaster monitoring small satellite. Next, we will improve contextual algorithm based on MODIS to adapt to HJ sensor.

\section{A. Brief introduction of environment and disaster monitoring small satellite}

The environment and disaster monitoring small satellite A, B satellites ( $\mathrm{HJ}-1 \mathrm{~A} / 1 \mathrm{~B}$ satellites) were launched successfully in Taiyuan Satellite Launch Center in China in September 6, 2008. Through trial operation of nearly 7 months, HJ-1A/1B were used formally and users can receive freely data. The HJ-1A/1B are two optical satellites of environment and disaster monitoring small satellite constellation that includes three satellites. The constellation's main task is to dynamically monitor natural disasters, ecological destruction and environmental pollution in large-scale, all-weather and all-time, predict change trend of disaster and ecological environment, assess condition of disaster and quality of environment rapidly and scientifically, improve ability of observation, collection, transmission and processing for information of disaster and environment, and provide scientific basis for emergency rescue, disaster relief and reconstruction and environmental protection.

The two optical satellites of HJ can provide optical remote sensing information of visible and infrared spectrum bands and have ability of quickly repeat detection with multi-spectral resolution, high spectral resolution and large field. The two optical small satellites can observe earth completely in two days, which monitor truly disaster and environment all-weather and long-term. HJ-1-A satellite carries two CCD cameras and a hyperspectral imager (HSI) and HJ-1-B satellite carries two CCD cameras and an infrared camera (IRS). The design principles of four CCD cameras are exactly same and are placed symmetrically to the subastral point, which divide equally field of observation, observe side by side and pushbroom imaging jointly which its ground swath width is $700 \mathrm{~km}$, the resolution of ground pixels is 30 meters and includes four spectral bands. In addition, there is a hyperspectral imager carried by HJ-1-A satellite, which its ground swath width is $50 \mathrm{~km}$, the resolution of ground pixels is 100 meters, includes 110 128 spectral bands, has $\pm 30^{\circ}$ side observing ability and calibration function on satellite. There is an infrared camera carried by HJ-1-B satellite, which its ground swath width is
$720 \mathrm{~km}$, the resolution of ground pixels is 150 meters/300meters, includes four spectral bands such as near infrared, short infrared, middle infrared and thermal infrared.

The environment and disaster monitoring small satellite constellation has some properties such as high spatial resolution, high time resolution, high spectral resolution and wide observation width, which is a system composed of optical satellite and synthetic aperture radar satellite and can use visible light, infrared and microwave remote sensing observation facilities to meet observation demand of monitoring environment and forecasting disaster for time resolution, space resolution, spectral resolution and allweather, all-time. Thus, it is very likely to monitor forest fires by using environment and disaster monitoring small satellite which is invented independently by China.

\section{B. Comparing of HJ and MODIS data}

Although the infrared bands of HJ IRS are less than infrared bands of MODIS, it has $4 \mu \mathrm{m}$ and $11 \mu \mathrm{m}$ that can be used to detect fire spots. Although there are some differences in subsidiary bands, it can meet basically requirements of contextual algorithm based on MODIS. The process of improved fire spots detection algorithm includes potential fire spots judgment, absolute fire spots judgment, background characteristics analysis, relative fire spots judgment and fire spots confidence. It cannot remove false fire spots, because HJ data is different from MODIS data.

\section{Water and cloud mask}

The forest fire detection algorithm extracts water information by using land and water mask products of MOD03 from MODIS data. Cloud products extract cloud information by combining multiple bands based on cloud detection algorithm. HJ data products are different from MODIS data products, so we only can extract water and cloud information according to characteristics of HJ IRS data.

(1) Water mask

In general, we can extract water information using normalized difference water index (NDWI), NDWI $=(\mathrm{TM} 2$ - TM5)/ (TM2 + TM5), where TM2 band represents green band, TM5 band represents middle-infrared band. However, the HJ IRS sensor only has infrared bands, lack of green band; we cannot extract water information using NDWI. From reflectance spectral curve of water, we can see that reflectance of water in middle-infrared band $(1.65 \mu \mathrm{m})$ is very low, so we can extract water information only using middle-infrared band.

Through statistical analysis, we get judgment condition of water based on HJ IRS sensor, as follows:

$$
\text { Water }=(\text { Riance } 1.65<6) \text { and }\left(\mathrm{T}_{4}<272 \mathrm{~K}\right)
$$

Where, Water represents water information, Riance1.65 represents radiation brightness of infrared band that its center wavelength is $1.65 \mu \mathrm{m}, \mathrm{T}_{4}$ represents brightness temperature of infrared band that its center wavelength is $4 \mu \mathrm{m}$. 


\section{(2) Cloud mask}

The principle of cloud detection is that visible light band has strong reflectivity and infrared band temperature is low. Learning from cloud detection algorithm based on MODIS data, we use brightness temperature of infrared band that its center wavelength is $11 \mu \mathrm{m}$, through statistical analysis, and get judgment condition of cloud based on $\mathrm{HJ}$ IRS sensor, as follows:

$$
\text { Cloud }=\left(\mathrm{T}_{11}<265\right) \text { and }(\text { Water }=0)
$$

Where, Cloud represents cloud information, $T_{11}$ represents brightness temperature of infrared band that its center wavelength is $11 \mu \mathrm{m}$, Water represents water information.

\section{Potential fire spots judgment}

From statistical analysis, we get judgment threshold value of potential fire spots is $325 \mathrm{~K}$ in the daytime. If the brightness temperature of third band of pixels meet the formula: $\mathrm{T} 4>325 \mathrm{~K}$, these pixels are judged as potential fire spots.

\section{E. Absolute fire spots judgment}

We use the method of Kaufman to judge absolute fire spots, that is to say, if the brightness temperature of third band of pixels meet the formula: T4 $>360 \mathrm{~K}$, these pixels are judged as absolute fire spots.

\section{F. Background characteristics analysis}

We use adaptive window contextual spatial statistical method to judge potential fire spots pixels one by one in order to determine if it is absolute fire spots. The "effective background pixels" is a very important concept during conducting spatial statistic. The effective background pixels are pixels that take potential fire spots pixels as center and meet following four conditions: (1) Remote sensing data acquired are not spoiled data; (2) The pixels are land pixels; (3) The pixels are not cloud pixels; (4) The pixels are not background fire spots pixels that meet $\mathrm{T}_{4}>$ $325 \mathrm{~K}$ and $\mathrm{T}_{4}-\mathrm{T}_{11}>20 \mathrm{~K}$. A potential fire spot pixel is searched taking the potential fire spot pixel as center from $5 \times 5,7 \times 7$, to $21 \times 21$ window size; when the number of effective background pixels account for $25 \%$ of total pixels, the search should be stopped.

\section{G. Relative fire spots judgment}

After obtaining effective background pixels, we judge relative fire spots by using contextual spatial statistical method. The judgment conditions are as following in the daytime:

$$
\left[\mathrm{T}_{4}-\mathrm{T}_{11}>\mathrm{AVG}\left(\mathrm{T}_{4}-\mathrm{T}_{11}\right)+3.5 \delta_{(\mathrm{T} 4-\mathrm{T} 11)}\right] \text { and }\left[\left(\mathrm{T}_{4^{-}}\right.\right.
$$

$\left.\mathrm{T}_{11}\right)>\operatorname{AVG}\left(\mathrm{T}_{4}-\mathrm{T}_{11}\right)+6 \mathrm{~K}$ ] and $\left[\left(\mathrm{T}_{4}>\operatorname{AVG}\left(\mathrm{T}_{4}\right)\right)+3 \delta_{(\mathrm{T} 4)}\right]$ and

$$
\left\{\left[\mathrm{T}_{11}>\mathrm{AVG}_{\mathrm{T} 11}+\delta_{(\mathrm{T} 11)}-4 \mathrm{~K}\right] \text { or }\left[\delta^{\prime}{ }_{(\mathrm{T} 4)}>5 \mathrm{k}\right]\right\}
$$

Where, $\mathrm{AVG}_{(\mathrm{T} 4-\mathrm{T} 11)}$ represents mean of $\mathrm{T}_{4}-\mathrm{T}_{11}$ brightness temperature difference of effective background pixel, $\delta_{\text {(T4-T11) }}$ represents mean absolute deviation of $\mathrm{T}_{4}-\mathrm{T}_{11}$ brightness temperature difference of effective background pixel, $\operatorname{AVG}\left(\mathrm{T}_{4}\right)$ represents mean of $\mathrm{T}_{4}$ brightness temperature, $\operatorname{AVG}\left(\mathrm{T}_{11}\right)$ represents mean of $\mathrm{T}_{11}$ brightness temperature, $\delta_{(\mathrm{T} 4)}$ represents mean absolute deviation of $\mathrm{T}_{4}$ brightness temperature of effective background pixel, $\delta_{(\mathrm{T} 11)}$ represents mean absolute deviation of $\mathrm{T}_{11}$ brightness temperature of effective background pixel, $\delta^{\prime}{ }_{(\mathrm{T} 4)}$ represents mean absolute deviation of $\mathrm{T}_{4}$ brightness temperature of background fire spot pixel.

\section{H. Fire spots confidence}

The calculation of fire spots confidence of $\mathrm{HJ}$ is the same as MODIS.

\section{CONCLUSIONS}

In summary, the research of fire detection based on AVHRR AND MODIS has made many achievements. Especially, in recent years, a number of authors have focused on fire detection based on theoretical analysis, fixed threshold method, or contextual algorithms using NOAA Advanced Very High Resolution Radiometer (AVHRR) multi-channel data. Since the Moderate Resolution Imaging Spectroradiometer (MODIS) instruments onboard Terra and Aqua began collecting data in February 2000 (Terra) and June 2002 (Aqua), satellite fire detection capability has been improved using two $3.96 \mu \mathrm{m}$ channels. In addition, according to the characteristics of environment and disaster monitoring small satellite, the existing contextual algorithm based on MODIS is improved to adapt HJ-IRS. We believe that the fire detection algorithms based on remote sensing data will get more improvements in the future.

\section{ACKNOWLEDGMENT}

The study is supported by Henan Provincial Education Office Research Plan (2010B420001) and Fund of Henan Polytechnic University Young Teachers (Q2010-42).

\section{REFERENCES}

[1] Gao Maofang, Qin Zhihao, Liu Sanchao. A STUDY OF FOREST FIRE DETECTION BASED ON MODIS DATA [J]. Remote Sensing for Land \& Resources, 2005(2):60-63.

[2] Zhao Yingshi. Principle and Methods of Remote Sensing Application and Analysis [M]. Beijing: Science Press, 2003.

[3] Kaufman YJ, Justice CO, Flynn L P, eta1. Potential Global Fire Monitoring from EOS-MODIS [J]. Journal of Geophysical Research, 1998, (103): 32215-32238.

[4] Flasse SP, Ceccato P. A Contextual Algorithm for AVHRR fire detection [J]. INT. J. Remote Sensing, 1996 (17): 419-424.

[5] Chuvieco E. Martin M P. A Simple Method for Fire Growth Mapping Using AVHRR Channel 3 data [J]. INT. J. Remote Sensing, 1994(15): 3141-3146.

[6] Boles S H, Verbyla D L. Comparison of Three AVHRR-Based Fire Detection Algorithms for Interior Alaska [J]. Remote Sensing of Environment, 2000 (72):1-16.

[7] Dozier J. A method for satellite identification of surface temperature fields of subpixel resolution [J]. Remote Sensing of Environment. 1981 (11): 221-229.

[8] Qin Xianji, Yi Haoruo. A Method to Identify Forest Fire Based on MODIS Data [J]. FIRE SAFETY SCIENCE, 2004, 13(2): 83-89. 
[9] Liang Yun. Monitoring the Forest Fire by Using EOS/MODIS Data [J] REMOTE SENSING T ECHNOLOGY AND APPLICATION, 2002, 17(6): 310-312

[10] Liu Liangming, Yan Junjie. Fire Detection Based on EOS MODIS Data $[\mathrm{J}]$. Geomatics and Information Science of Wuhan University, 2004, 29(1): 55-58.

[11] Zhang Shuyu, Jing Yigang. Study on Application of EOS-MODIS Data to Forest Fire Monitoring [J]. Journal of Catastrophology, 2004, 19(1): 58-62.

[12] Zhou Xiaocheng, Wang Xiaoqin. Validation Analysis of the Algorithm for Identifying Forest Fire based on MODIS Data [J]. Fire Safety Science, 2006, 15(1): 31-37.

[13] Huang Jing, Xia Zhihong. The Application of EOS/MODIS Data in Monitor Forest Fire of Hubei Province [J]. Torrential Rain and Disasters, 2008, 27(2): 182-185.

[14] Peng Guangxiong, Shen Wei, Hu Deyong. Method to Identify Forest Fire Based on Smoke Plum ES Masking By Using MODIS Data [J]. J. Infrared Millim., 2008, 27(3):185-189.

[15] Peng Guangxiong, Chen Yunhao, Li Jing. Combination of Remote Sensing and Meteorological Data for Fire Risk Monitoring-A Case Study in Peninsular Malaysia [J]. GEO-INFORMATION SCIENCE, 2007, 9(5):99-104.

[16] Justice C O, Giglio L. Kaufman Y, eta1. The MODIS fire products [J] Remote Sensing of Environment, 2002(83): 244-262.

[17] Giglio L, Descloitres J, Justice C O, eta1. An Enhanced Contextual Fire Detection Algorithm for MODIS [J]. Remote Sensing of Environment. 2003(87): 273-282.
[18] Qin Xianji, Zhang Zihui, Li Zengyuan. An Automatic Forest Fires Identification Method Using HJ-1B IRS Data [J]. REMOTE SENSING TECHNOLOGY AND APLICATION, 2010, 25(5): 700705 .

[19] XIE Qi-Yuan, ZHANG He-Ping, ZHANG Yong-Ming, etal. Experimental study on stokes scattering matrixes of smoke particles [J]. Infrared Millim. Waves. 2007, 26(4): 279-283.

[20] Xie Y, Qu J, Hao X. Smoke plume detecting using MODIS measurements in eastern United States[C]. East FIRE Conference Proceedings, Fairfax, VA, 2005, May 11-13.

[21] Louis Giglio, Jacques Descloitres. An Enhanced Contextual Fire Detection Algorithm for MODIS [J]. Remote Sensing of Environment, 2003(87): 273-282.

[22] Todd J. Hawbaker, Volker C. Detection rates of the MODIS active fire product in the United States [J]. Remote Sensing of Environment, 2008(112): 2656-2664.

[23] Wanting Wang, John J. Qu. An improved algorithm for small and cool fire detection using MODIS data: A preliminary study in the southeastern United States [J]. Remote Sensing of Environment, 2007(128): 163-170.

[24] Wilfrid Schroeder, Elaine Prins. Validation of GOES and MODIS active fire detection products using ASTER and ETM+ data [J]. Remote Sensing of Environment, 2008(112): 2711-2726.

[25] D.P. Roy, Y. Jin. Prototyping a global algorithm for systematic fireaffected area mapping using MODIS time series data $[\mathrm{J}]$. Remote Sensing of Environment, 2005(97): 137-162. 